\title{
Two planets orbiting the recently formed post-common envelope binary NN Serpentis ${ }^{\star}$
}

\author{
K. Beuermann ${ }^{1}$, F. V. Hessman ${ }^{1}$, S. Dreizler ${ }^{1}$, T. R. Marsh ${ }^{2}$, S. G. Parsons ${ }^{2}$, D. E. Winget ${ }^{3}$, G. F. Miller ${ }^{3}$, \\ M. R. Schreiber ${ }^{4}$, W. Kley ${ }^{5}$, V. S. Dhillon ${ }^{6}$, S. P. Littlefair ${ }^{6}$, C. M. Copperwheat ${ }^{2}$, and J. J. Hermes ${ }^{3}$ \\ ${ }^{1}$ Institut für Astrophysik, Georg-August-Universität, Friedrich-Hund-Platz 1, 37077 Göttingen, Germany \\ e-mail: beuermann@astro.physik.uni-goettingen.de \\ 2 Department of Physics, University of Warwick, Coventry, CV4 7AL, UK \\ 3 Dept. of Astronomy, University of Texas at Austin, RLM 16.236, Austin, TX 78712, USA \\ 4 Departamento de Fisica y Astronomia, Universidad de Valparaiso, Av. Gran Bretana 1111, Valparaiso, Chile \\ ${ }^{5}$ Institut für Astronomie \& Astrophysik, Universität Tübingen, Morgenstelle 10, 72076 Tübingen, Germany \\ ${ }^{6}$ Department of Physics \& Astronomy, University of Sheffield, S3 7RH, UK
}

Received 26 July 2010 / Accepted 6 October 2010

ABSTRACT

\begin{abstract}
Planets orbiting post-common envelope binaries provide fundamental information on planet formation and evolution. We searched for such planets in NN Ser ab, an eclipsing short-period binary that shows long-term eclipse time variations. Using published, reanalysed, and new mid-eclipse times of NN Ser ab obtained between 1988 and 2010, we find excellent agreement with the light-travel-time effect produced by two additional bodies superposed on the linear ephemeris of the binary. Our multi-parameter fits accompanied by $N$-body simulations yield a best fit for the objects NN Ser $(\mathrm{ab}) \mathrm{c}$ and d locked in the 2:1 mean motion resonance, with orbital periods $P_{\mathrm{c}} \simeq 15.5$ yrs and $P_{\mathrm{d}} \simeq 7.7$ yrs, masses $M_{\mathrm{c}} \sin i_{\mathrm{c}} \simeq 6.9 M_{\text {Jup }}$ and $M_{\mathrm{d}} \sin i_{\mathrm{d}} \simeq 2.2 M_{\text {Jup }}$, and eccentricities $e_{\mathrm{c}} \simeq 0$ and $e_{\mathrm{d}} \simeq 0.20$. A secondary $\chi^{2}$ minimum corresponds to an alternative solution with a period ratio of 5:2. We estimate that the progenitor binary consisted of an A star with $\sim 2 M_{\odot}$ and the present $\mathrm{M}$ dwarf secondary at an orbital separation of $\sim 1.5 \mathrm{AU}$. The survival of two planets through the common-envelope phase that created the present white dwarf requires fine tuning between the gravitational force and the drag force experienced by them in the expanding envelope. The alternative is a second-generation origin in a circumbinary disk created at the end of this phase. In that case, the planets would be extremely young with ages not exceeding the cooling age of the white dwarf of $10^{6}$ yrs.
\end{abstract}

Key words. binaries: eclipsing - planetary systems - stars: individual: NN Ser - novae - cataclysmic variables planets and satellites: formation - stars: evolution

\section{Introduction}

NN Ser ab ${ }^{1}$ is a short-period $\left(P_{\text {orb }}=3.12 \mathrm{~h}\right)$ eclipsing binary at a distance of $500 \mathrm{pc}$. The detached system contains a hot hydrogen-rich white dwarf NNSera of spectral type DAO1 and an M4 dwarf star NN Serb with masses of $0.535 M_{\odot}$ and $0.111 M_{\odot}$, respectively (Parsons et al. 2010a). With an effective temperature of $57000 \mathrm{~K}$ (Haefner et al. 2004), the white dwarf has a cooling age of only $10^{6}$ yrs (Wood 1995). The present system resulted from a normal binary with a period of $\sim 1$ year when the more massive component evolved to a giant and engulfed the orbit of its companion. The subsequent common envelope (CE) phase led to the expulsion of the envelope, laying bare the newly born white dwarf and substantially shortening the orbital period.

Some eclipsing post-CE binaries display long-term eclipse time variations, among them V471 Tau (Kamiński et al. 2007), QS Vir and NN Ser (Parsons et al. 2010b, and references therein). The latter possesses deep and well-defined eclipses, which allow measurements of the mid-eclipse times to an accuracy of

^ Table 3 is only available in electronic form at http: //www . aanda. org

${ }^{1}$ On recommendation by the Editor of A\&A, we refer to the system as NN Ser, to the binary explicitly as NN Ser ab, and to the objects orbiting the binary as NN Ser (ab)c and NN Ser (ab)d.
$100 \mathrm{~ms}$ and better (Brinkworth et al. 2006; Parsons et al. 2010b). The processes advanced to explain them include the long-term angular momentum loss by gravitational radiation and magnetic braking, possible quasi-periodicities caused, e.g., by Applegate's (1992) mechanism, and the strict periodicities produced by apsidal motion or the presence of a third body in the system. Finding the correct interpretation requires measurements of high precision and a coordinated effort over a wide range of time scales. The existence of a third body orbiting NN Ser ab was previously considered by Qian et al. (2009), but the orbital parameters suggested by them are incompatible with more recent data (Parsons et al. 2010b). In this Letter, we present an analysis of the eclipse time variations of NN Ser ab, based on published data, the reanalysis of published data, and new measurements obtained over the first half of 2010.

\section{The data}

After their 1988 discovery of deep eclipses in NN Ser, Haefner et al. (2004) acquired a series of accurate mid-eclipse times in 1989. After a hiatus of ten years, they added a potentially very accurate trailed CCD imaging observation using the ESO VLT. From 2002 on, the Warwick group systematically secured a total of 22 mid-eclipse times of high precision 
Table 1. Revised and new eclipse times of NN Ser ab with residuals for the light-travel-time effect produced by the two planets of Model $2 \mathrm{a}^{1}$.

\begin{tabular}{|c|c|c|c|c|}
\hline \multirow[t]{2}{*}{$\mathrm{E}$} & \multirow{2}{*}{$\begin{array}{c}\text { BJD(TT) } \\
\text { JD2450000+ }\end{array}$} & \multicolumn{2}{|c|}{ Error } & \multirow{2}{*}{$\begin{array}{c}\text { Residual } \\
\text { (s) }\end{array}$} \\
\hline & & (days) & (s) & \\
\hline \multicolumn{5}{|c|}{ (a) Revised, VLT $V$-band and Wroclaw Obs. white light: } \\
\hline 30721 & 1340.7165402 & 0.0000023 & 0.20 & -0.03 \\
\hline 33233 & 1667.4780058 & 0.0000960 & 8.29 & 0.35 \\
\hline \multicolumn{5}{|c|}{ (b) MONET/North 1.2-m white light photometry: } \\
\hline 60489 & 5212.9418187 & 0.0000069 & 0.60 & 0.23 \\
\hline 60505 & 5215.0230961 & 0.0000066 & 0.57 & -0.15 \\
\hline 60528 & 5218.0149380 & 0.0000043 & 0.37 & -0.21 \\
\hline 60735 & 5244.9415254 & 0.0000029 & 0.25 & 0.10 \\
\hline 60743 & 5245.9821654 & 0.0000032 & 0.28 & 0.03 \\
\hline 60751 & 5247.0228063 & 0.0000034 & 0.29 & 0.02 \\
\hline 60774 & 5250.0146469 & 0.0000034 & 0.29 & -0.16 \\
\hline \multicolumn{5}{|c|}{ (c) McDonald 2.1-m photometry with Schott $B G 40$ filter: } \\
\hline 60927 & 5269.9169047 & 0.0000014 & 0.12 & -0.16 \\
\hline 60950 & 5272.9087487 & 0.0000013 & 0.11 & -0.04 \\
\hline 61426 & 5334.8268834 & 0.0000018 & 0.16 & -0.22 \\
\hline 61440 & 5336.6480059 & 0.0000018 & 0.16 & -0.15 \\
\hline 61441 & 5336.7780894 & 0.0000015 & 0.13 & 0.15 \\
\hline 61564 & 5352.7779443 & 0.0000016 & 0.14 & 0.15 \\
\hline \multicolumn{5}{|c|}{ (c) ESO NTT 3.5-m ULTRACAM Sloan $g^{\prime}$ photometry: } \\
\hline 61219 & 5307.9003015 & 0.0000010 & 0.09 & 0.04 \\
\hline 61579 & 5354.7291448 & 0.0000009 & 0.08 & 0.03 \\
\hline
\end{tabular}

Notes. ${ }^{(1)}$ Table 3 in the electronic version of the journal provides a list of all eclipse times used in this work.

(Brinkworth et al. 2006; Parsons et al. 2010b, this work). Parsons et al. (2010b) list all published mid-eclipse times by other authors until the end of 2009. These are included in our analysis that weights them by their statistical errors. Since the individual Warwick mid-eclipse times between 2002 and 2009 were separated by about one year, information on eclipse time variations on a shorter time scale is lacking. We, therefore, organized a collaborative effort of the Göttingen, McDonald, and Warwick groups to monitor NN Ser over the first half of 2010. We used the remotely controlled MONET/North 1.2-m telescope at McDonald Observatory via the MONET internet remote-observing interface, the McDonald 2.1-m telescope, and the ESO 3.5-m NTT. The MONET data were taken in white light, the McDonald data with a $B G 40$ filter, and the NTT observations were acquired with the ULTRACAM high-speed CCD camera equipped with Sloan filters. The mid-eclipse times measured in Sloan $u^{\prime}, g^{\prime}$, and $i^{\prime}$ are consistent, and we used the $g^{\prime}$ data as the most accurate set for the present purpose. Table 1 lists the new mid-eclipse times shifted to the solar system barycenter and corrected for leap seconds. The table also gives the 1- $\sigma$ statistical errors and the residuals relative to our final fit shown in Fig. 2 and discussed in Sect. 4, below.

The mid-eclipse time derived by Haefner et al. (2004) from the trailed VLT image of 11 June $1999^{2}$ is the most variant of the published eclipse time measurements and was assigned a large error of $17 \mathrm{~s}$, although this should be a very precise measurement, given the very simple form of the eclipses in NN Ser and the use of an $8.2 \mathrm{~m}$ telescope. We reanalysed the image of 11 June 1999, which started 04:53:05.537 UT with an exposure of $1125.7462 \mathrm{~s}$ and was taken in good atmospheric conditions. The key issue is the conversion of the track from pixel space to time. Using two independent methods, we found that the original analysis by Haefner et al. (2004) was in error and that the

\footnotetext{
2 http://www.eso.org/public/images/eso9936b/
}
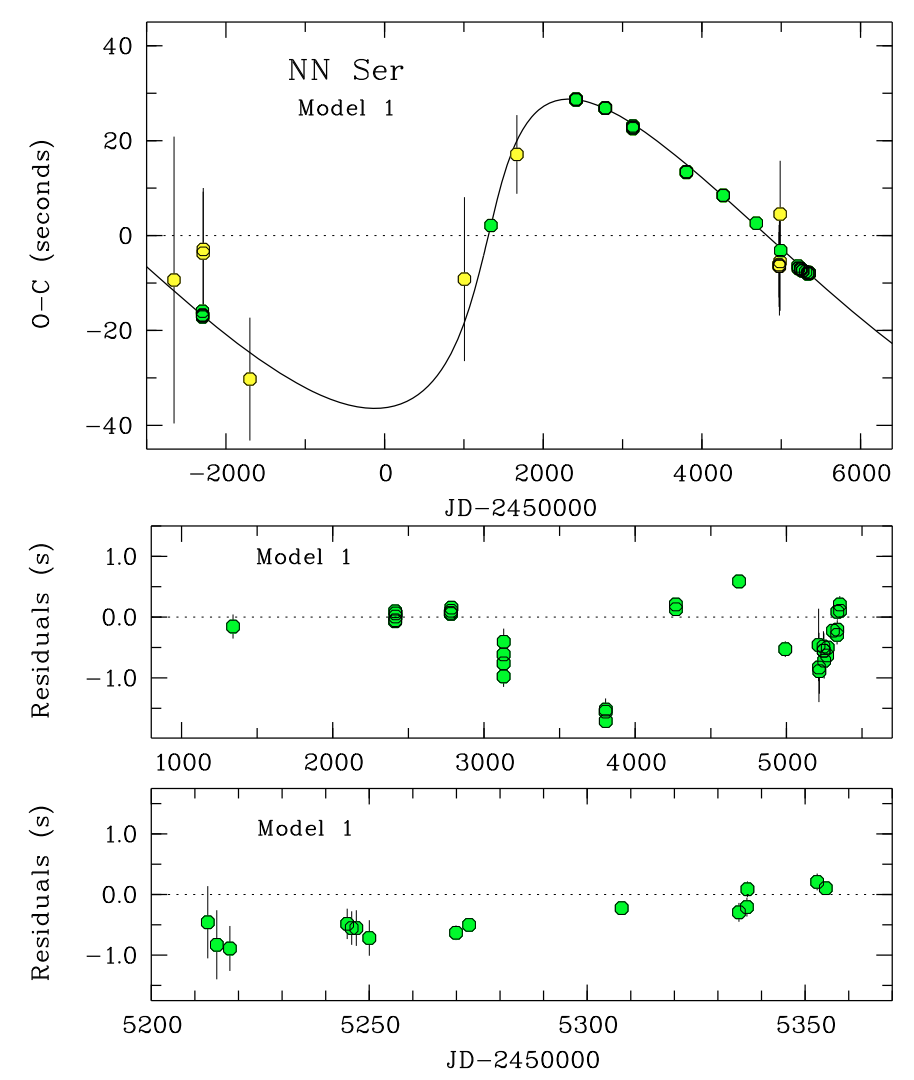

Fig. 1. Top: observed - calculated mid eclipse time differences relative to the best-fit linear ephemeris for Model 1 of a single planet orbiting NN Ser ab. Center and bottom: residuals relative to the eccentric-orbit fit for two selected time intervals.

mid-eclipse time can be determined with an accuracy of $0.20 \mathrm{~s}$ (Table 1 , cycle $E=30721$ ). We also reanalysed the less accurate data of Pigulski \& Michalska (2002) (cycle $E=33233$ ) by including the effects of the finite integration times.

\section{The light-travel-time effect in NN Ser}

All measurements of mid-eclipse times of NNSer ab are displayed in Figs. 1 and 2 as $\mathrm{O}-\mathrm{C}$ values relative to the modeldependent linear ephemerides of the respective fits. Data points with errors $<1 \mathrm{~s}$ and $>1 \mathrm{~s}$ are shown as green and yellow dots, respectively. The eclipse time measurements dominating the fit are the 1989 data points of Haefner et al. (2004) near the abscissa value $\mathrm{JD}^{\prime}=\mathrm{JD}-2450000=-2295$, the reevaluated VLT point on $\mathrm{JD}^{\prime}=1340$, the 2002-2009 series of Warwick eclipse times since $\mathrm{JD}^{\prime}=2411$ (Parsons et al. 2010b), and the data of this work since $\mathrm{JD}^{\prime}=5212$. In particular, the revised VLT mideclipse time implies a twofold change in the time derivative of $\mathrm{O}-\mathrm{C}$ and excludes the simple quadratic ephemerides used by Brinkworth et al. (2006) and Parsons et al. (2010b). The available data do not exclude abrupt period changes or an ultimate aperiodicity, but there is no physical process that predicts such behavior. We consider a periodic behavior the most promising assumption and proceed to explore this possibility.

Strictly periodic O-C variations may result from apsidal motion of the binary orbit or an additional body orbiting the binary. Given the parameters of NN Ser ab, classical apsidal motion for small eccentricities $e_{\text {bin }}$ produces a sinusoidally varying time shift with an amplitude $P_{\text {bin }} e_{\text {bin }} / \pi=3577 e_{\text {bin }}$ s (Todoran 1972). As a result, $e_{\text {bin }} \sim 0.01$ would suffice to produce the observed amplitude. However, the likewise predicted variation 

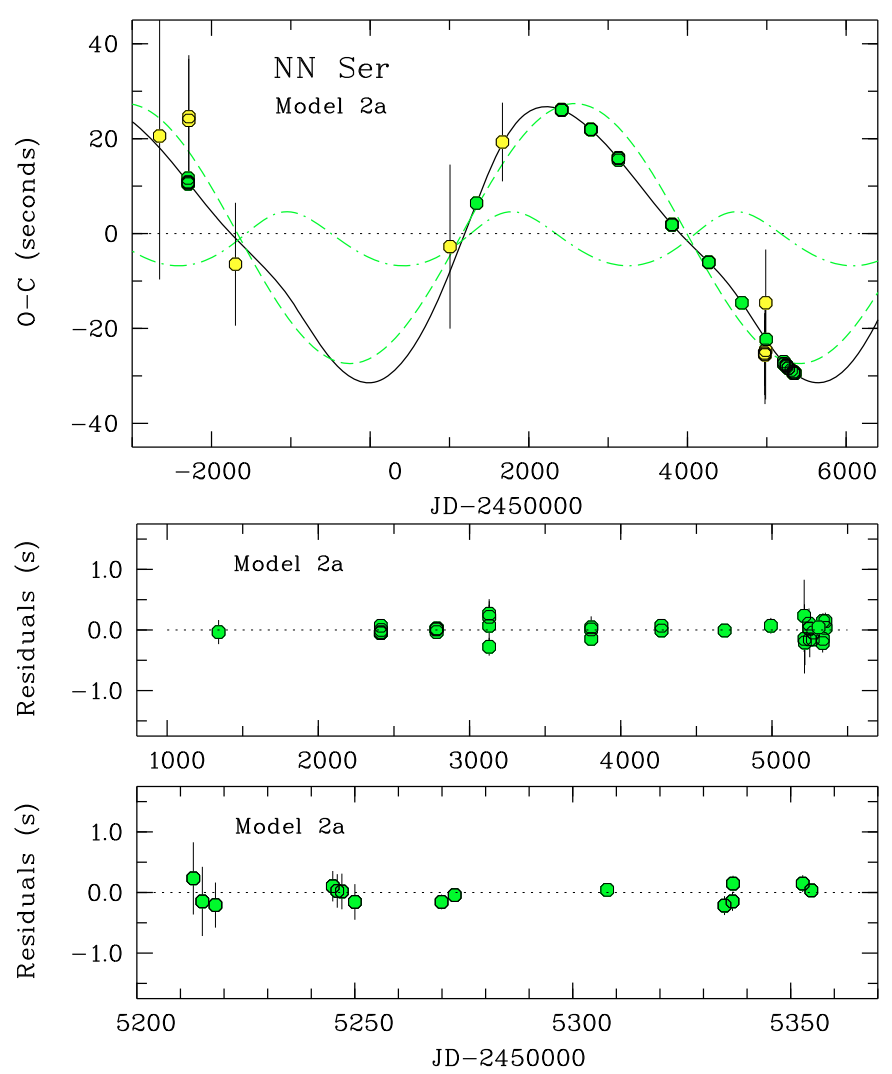

Fig. 2. Same as Fig. 1 for Model 2a of two planets orbiting NN Ser ab. The contributions of components $\mathrm{b}$ and $\mathrm{c}$ are indicated by the dashed and dot-dashed curves, respectively, the solid curve shows the combined effect.

of the FWHM of the eclipse and the time shift of the secondary eclipse are not observed (Parsons et al. 2010b, this work). Furthermore, the observed variation is not sinusoidal and, given an apsidal motion constant for the secondary star NN Ser b of $k_{22} \simeq 0.11$, the period of the apsidal motion would be as short as $\sim 0.4$ years. Such periodicity is not detected (see Fig. 2, bottom panel).

This leaves us with the third-body hypothesis, at least for the major fraction of the observed eclipse time variations. In general, it would be possible that different physical processes combine to produce the observed signal. We find, however, that a perfect fit within the very small statistical errors can be obtained for a signal that consists of the periodicities produced by two objects orbiting NN Ser ab. Guided by Ockham's razor and the history of discoveries in the Solar system, we consider that a fourth body in the presence of a third one is a natural assumption.

\section{One-planet and two-planet fits to the data}

Including the light-travel-time effect of the objects NN Ser (ab)c and NN Ser $(a b) d$, the times of mid-eclipse become

$T=T_{0}+P_{\mathrm{bin}} E+\sum_{\mathrm{k}=\mathrm{c}, \mathrm{d}} \frac{K_{\mathrm{bin}, \mathrm{k}}\left(1-e_{\mathrm{k}}^{2}\right)}{\left(1+e_{\mathrm{k}} \cos v_{\mathrm{k}}\right)} \sin \left(v_{\mathrm{k}}-\varpi_{\mathrm{k}}\right)$,

where time is measured from a fiducial mid-eclipse time $T_{0}$. A linear binary ephemeris is assumed with $P_{\text {bin }}$ the orbital period and $E$ the cycle number. The five free parameters for planet $k$ are the orbital period $P_{\mathrm{k}}$, the eccentricity $e_{\mathrm{k}}$, the longitude of periastron $\varpi_{\text {bin,k }}$ measured from the ascending node in the plane of the sky, the time $T_{\mathrm{k}}$ of periastron passage, and the amplitude of the eclipse time variation $K_{\mathrm{k}}=a_{\mathrm{bin}, \mathrm{k}} \sin i_{\mathrm{k}} / \mathrm{c}$, with $a_{\mathrm{bin}, \mathrm{k}}$ the semimajor axis of the orbit of the center of mass of the binary about the common center of mass of the system, $i_{\mathrm{k}}$ the inclination, and $\mathrm{c}$ the speed of light. In the denominator, $v_{\mathrm{k}}$ is the true anomaly, which progresses through $2 \pi$ over the orbital period $P_{\mathrm{k}}$.

We explored the multi-dimensional $\chi^{2}$ space of the twoplanet model, using the Levenberg-Marquardt routine implemented in IDL and an independent code. The search showed that compensation effects render some parameters ill defined. This uncertainty results, in particular, from the long hiatus between the accurate measurements of 1989 (Haefner et al. 2004) and 1999 (VLT, this work). We selected the best model, therefore, by imposing the additional requirement that the derived orbits be secularly stable. We investigated all solutions permitted by the data with numerical $N$-body simulations with a variable time step Runge-Kutta integrator, following the orbits over $10^{5} \mathrm{yrs}$, and find that only a narrow range in parameter space corresponds to stable solutions. In what follows, we consider the one-planet and the two-planet models in turn.

Model 1 with seven free parameters describes a single planet with eccentricity $e$. The fit requires $e \gtrsim 0.60$ and is bad for any value of $e$, with a reduced $\chi_{v}^{2} \geq 23.3\left(\chi^{2}=1052\right.$ for 45 degrees of freedom). The top panel of Fig. 1 shows the case $e=0.65$. The residuals based on the statistical errors of the data points (center panel) reach 23 standard deviations and indicate that there is an additional modulation at about half the orbital period. The residuals of the 2010 data (bottom panel) demonstrate the lack of $\mathrm{O}-\mathrm{C}$ fluctuations on a short time scale.

Model 2 for two planets requires some restriction in parameters, because the grid search yields good fits for a range of eccentricities of the outer planet $e_{\mathrm{c}}$, including zero, and for a period ratio $r_{\mathrm{p}}=P_{\mathrm{c}} / P_{\mathrm{d}}=1.90 \pm 0.30$ or $r_{\mathrm{p}}=2.50 \pm 0.15(1-\sigma$ errors), with the former slightly preferred. The dichotomy in $r_{\mathrm{p}}$ arises from the uncertain phasing of the singular 1989 point relative to the train of the 1999-2010 data. Further minima at still larger $r_{\mathrm{p}}$ do not exist. Only a small fraction of the parameter space allowed by the fits corresponds to secularly stable orbits, however. Near $r_{\mathrm{p}} \simeq 2$, orbits with $e_{\mathrm{c}}>0.1$ tend to be unstable, while the stability region is broad in the remaining parameters for $e_{\mathrm{c}} \approx 0.02$. Furthermore, all solutions with $r_{\mathrm{p}} \lesssim 1.9$ are unstable, with only some solutions stable at $r_{\mathrm{p}}=1.9$. The solutions near $r_{\mathrm{p}}=2.5$ are more generally stable. We consider Models $2 \mathrm{a}$ and $2 \mathrm{~b}$, representing the cases of $r_{\mathrm{p}} \simeq 2.0$ and 2.5, respectively, both with $e_{\mathrm{c}} \equiv 0$. Model 2a provides the slightly better fit and is shown in Fig. 2 . It yields $K_{\mathrm{c}}=27.4 \mathrm{~s}$, $K_{\mathrm{d}}=5.7 \mathrm{~s}, P_{\mathrm{c}}=15.5 \mathrm{yrs}, P_{\mathrm{d}}=7.75 \mathrm{yrs}$, and $e_{\mathrm{d}}=0.20$ with $\chi_{v}^{2}=0.78\left(\chi^{2}=32.9\right.$ for 42 d.o.f. $)$. Periastron passage of $\mathrm{NN} \operatorname{Ser}(\mathrm{ab}) \mathrm{d}$ occurred last on $\mathrm{JD}^{\prime} \simeq 4515$. At that time NNSer $(\mathrm{ab}) \mathrm{c}$ was at longitude $213^{\circ}$. For the low value of $e_{\mathrm{c}}=$ 0.03 , a shallow minimum of $\chi^{2}$ is attained for aligned apses. From the present data, we cannot infer the true value of $r_{\mathrm{p}}$ with certainty, but it is intriguing that objects $\mathrm{c}$ and $\mathrm{d}$ may be locked in either the 2:1 resonance, found also in other planetary systems, or the 5:2 resonance. The parameters for Models $2 \mathrm{a}$ and $2 \mathrm{~b}$ are listed in Table 2, together with their 1- $\sigma$ errors. A simpler model with two circular orbits reaches only $\chi_{v}^{2}=1.96\left(\chi^{2}=86.2\right.$ for 44 d.o.f.) at $r_{\mathrm{p}}=2.46$ and can be excluded.

Using Model 2a as input to our $N$-body simulations, we find that $e_{\mathrm{c}}$ and $e_{\mathrm{d}}$ oscillate around 0.02 and 0.22 with amplitudes of 0.02 and 0.05 , respectively. The difference $\Delta \varpi$ of the periastron longitudes circulates on a time scale of $400 \mathrm{yrs}$. The periods perform small-amplitude anti-phased oscillations, which cause $r_{\mathrm{p}}$ to oscillate between 1.9 and 2.2. Even if the two planets are secularly locked in the 2:1 mean motion resonance, therefore, 
Table 2. Parameters of the models fitted to the measured mid-eclipse time variations of NN Ser, where $\equiv$ indicates a fixed parameter.

\begin{tabular}{|c|c|c|c|c|c|c|c|c|c|c|c|c|c|c|c|}
\hline Model & Planets & $\begin{array}{l}\text { Number } \\
\text { free par. }\end{array}$ & $\begin{array}{c}P_{\mathrm{c}} \\
(\mathrm{yrs})\end{array}$ & $\begin{array}{c}P_{\mathrm{d}} \\
(\mathrm{yrs})\end{array}$ & $P_{\mathrm{c}} / P_{\mathrm{d}}$ & $e_{\mathrm{c}}$ & $e_{\mathrm{d}}$ & $\begin{array}{c}a_{\mathrm{c}} \\
(\mathrm{AU})\end{array}$ & $\begin{array}{c}a_{\mathrm{d}} \\
(\mathrm{AU})\end{array}$ & $\begin{array}{l}\varpi_{\mathrm{c}} \\
\left(^{\circ}\right)\end{array}$ & $\begin{array}{l}\varpi_{\mathrm{d}} \\
\left(^{\circ}\right)\end{array}$ & $\begin{array}{c}M_{\mathrm{c}} \sin i_{\mathrm{c}} \\
\left(M_{\mathrm{Jup}}\right)\end{array}$ & $\begin{array}{c}M_{\mathrm{d}} \sin i_{\mathrm{d}} \\
\left(M_{\mathrm{Jup}}\right)\end{array}$ & $\chi^{2}$ & $\chi_{v}^{2}$ \\
\hline 1 & 1 & $2+5$ & 22.60 & & & $\gtrsim 0.65$ & & 6.91 & & 8.0 & & 8.36 & & 1052.3 & 23.38 \\
\hline $2 a$ & 2 & $2+8$ & 15.50 & 7.75 & 2.00 & $\equiv 0.0$ & 0.20 & 5.38 & 3.39 & & 74 & 6.91 & 2.28 & 32.9 & 0.78 \\
\hline & & & \pm 0.45 & \pm 0.35 & \pm 0.15 & & \pm 0.02 & \pm 0.20 & \pm 0.10 & & \pm 4 & \pm 0.54 & \pm 0.38 & & \\
\hline $2 b$ & 2 & $2+8$ & $\begin{array}{r}16.73 \\
\pm 0.26\end{array}$ & $\begin{array}{r}6.69 \\
\pm 0.40\end{array}$ & $\begin{array}{r}2.50 \\
\pm 0.15\end{array}$ & $\equiv 0.0$ & $\begin{array}{r}0.23 \\
\pm 0.04\end{array}$ & $\begin{array}{r}5.66 \\
\pm 0.06\end{array}$ & $\begin{array}{r}3.07 \\
\pm 0.13\end{array}$ & & $\begin{array}{r}73 \\
\pm 7\end{array}$ & $\begin{array}{r}5.92 \\
\pm 0.40\end{array}$ & $\begin{array}{r}1.60 \\
\pm 0.27\end{array}$ & 33.8 & 0.80 \\
\hline
\end{tabular}

the observed period ratio at any given time may deviate slightly from its nominal value.

For Model 2a, the best-fit binary ephemeris is $T=$ BJED $2447344.524425(40)+0.1300801419(10) E$, where the errors refer to the last digits. Adding a quadratic term $B E^{2}$ to the ephemeris does not improve the two-planet fit and yields a $1-\sigma$ limit of $|B|<1.5 \times 10^{-13}$ days, leaving room for a period change by gravitational radiation or a long-term activity-related effect (Brinkworth et al. 2006; Parsons et al. 2010b).

\section{Discussion}

The large amplitude of the O-C eclipse time variations in NN Ser can only be explained by a third body in the system, while the still substantial residuals from a single-planet fit could, in principle, have a different origin from that of a fourth body. The twoplanet model, however, possesses the beauty of simplicity, and the fact that the residuals for the entire data set vanish simultaneously imposes tight restrictions on any other mechanism. In particular, the lack of short-term variability of the residuals in the first half of 2010 argues against any process that acts on a short time scale or leads to erratic eclipse time variations. Hence, there is strong evidence for two planets orbiting NN Ser ab.

With masses $M_{\mathrm{c}} \sin i_{\mathrm{c}} \simeq 6 M_{\text {Jup }}$ and $M_{\mathrm{d}} \sin i_{\mathrm{d}} \simeq 2 M_{\text {Jup }}$, NN Ser (ab)c and NN Ser (ab)d both qualify as giant planets for all inclinations $i_{\mathrm{c}}>28^{\circ}$ and $i_{\mathrm{d}}>9^{\circ}$, respectively. The probable detection of resonant motion with a period ratio of either $2: 1$ or 5:2 is a major bonus, which adds to the credence of the twoplanet model. It is the second planetary system found by eclipse timing, after HW Vir (Lee et al. 2009).

Given a pair of planets orbiting a post-CE binary, two formation scenarios are possible. They could either be old firstgeneration planets that formed in a circumbinary protoplanetary disk or they could be young second-generation planets formed $\$ 10^{6}$ yrs ago in a disk that resulted from the CE (Perets 2010). To evaluate both scenarios, we have reconstructed the CE evolution of NN Ser ab using the improved algorithm by Zorotovic et al. (2010), who constrain the CE efficiency to a range $\alpha \simeq 0.2-0.3$. Possible solutions for the progenitor binary of NN Ser ab are not very sensitive to $\alpha$ : for $\alpha=0.25$, the progenitor was a giant of $2.08 M_{\odot}$ and radius $194 R_{\odot}$ with the present secondary star at a separation of $1.44 \mathrm{AU}$. When the CE engulfed the secondary star, dynamic friction caused the latter to spiral in rapidly, thereby dramatically decreasing the binary separation to the current $0.0043 \mathrm{AU}$. Stability arguments imply that any planet from the pre-CE phase must have formed with semi-major axes exceeding 3.5 AU (Holman \& Wiegert 1999). With three quarters of the central mass expelled in the CE event, pre-existing planets would move outward or may even be lost from the system. However, given a sufficiently dense and slowly expanding CE, the dynamical force experienced by them may have ultimately moved them inward (Alexander et al. 1976). Since the drag primarily affects the more massive and more slowly moving outer planet, such a scenario could lead to resonant orbits, so a first-generation origin appears possible.

The alternative post-CE origin in a second-generation of planet formation is also possible, since the formation of circumbinary disks is a common phenomenon among post-AGB binary stars and the concentration of a slow, dusty wind to the orbital plane of the binary is thought to favor the formation of planets (e.g. van Winckel et al. 2009; Perets 2010). In particular the tiny separation of the present binary poses no problem for stable orbits of second-generation planets even at significantly shorter distances than the inner planet that we have detected (Holman \& Wiegert 1999). A particularly intriguing aspect of a second-generation origin of the planets in NN Ser would be their extreme youth, equal to or less than the $10^{6}$ yrs cooling age of the white dwarf (Wood 1995). This feature would distinguish them from all known exoplanets and may ultimately lead to their direct detection. While we cannot presently prove a second-generation origin for these planets, modeling the $\mathrm{CE}$ event may allow us to distinguish between the two scenarios.

Acknowledgements. We would like to thank Dr. Reinhold Haefner for information concerning the original VLT observations and analyses and Dr. Andrzej Pigulski for sending us his original photometry. This work is based on data obtained with the MONET telescopes funded by the "Astronomie \& Internet" program of the Alfried Krupp von Bohlen und Halbach Foundation, Essen, on observations with the ESO NTT under ESO programme 085.D-0541, and on data obtained from the ESO/ST-ECF Science Archive Facility. T.R.M., V.S.D., C.M.C., and S.P.L. acknowledge grant support from the UK's STFC. M.R.S. acknowledges support from FONDECYT under grant number 1061199 (MRS) and the Centre of Astrophysics Valparaíso. D.E.W. acknowledges the support of the Norman Hackerman Advanced Research Program under grant 003658-02552007.

\section{References}

Alexander, M. E., Chau, W. Y., \& Henriksen, R. N. 1976, ApJ, 204, 879 Applegate, J. H. 1992, ApJ, 385, 621

Brinkworth, C. S., Marsh, T., Dhillon, V. S., \& Knigge, C. 2006, MNRAS, 365,287

Haefner, R., Fiedler, A., Butler, K., \& Barwig, H. 2004, A\&A, 428, 181

Holman, M. J., \& Wiegert, P. A. 1999, AJ, 117, 621

Kamiński, K. Z., Ruciński, S. M., Matthews, J. M., et al. 2007, AJ, 134, 1206

Lee, J. W., Kim, S.-L., Kim, C.-H., et al. 2009, AJ, 137, 3181

Parsons, S. G., Marsh, T. R., Copperwheat, C. M., et al. 2010a, MNRAS, 402, 2591

Parsons, S. G., Marsh, T. R., Copperwheat, C. M., et al. 2010b, MNRAS, 407, 2362

Perets, H. B. 2010, ApJ, submitted [arXiv: 1001.0581$]$

Pigulski, A., \& Michalska, G. 2002, IBVS, 5218, 1

Qian, S.-B., Dai, Z.-B., Liao, W.-P., et al. 2009, ApJ, 706, L96

Todoran, I. 1972, Ap\&SS, 15, 229

van Winckel, H., Lloyd Evans, T., Briquet, M., et al. 2009, A\&A, 505, 1221

Wood, J. H., \& Marsh, T. R. 1991, ApJ, 381, 561

Wood, M. A. 1995, in White Dwarfs, ed. D. Koester, \& K. Werner (Heidelberg: Springer), LNP No. 443, 41

Zorotovic, M., Schreiber, M. R., Gänsicke, B. T., \& Nebot Gómez-Morán, A. 2010, A\&A, 520, 86 
Table 3. Previously published, reanalysed, and new mid-eclipse times of the white dwarf in NN Ser with residuals for the light-travel-time effect produced by the two planets of Model $2 \mathrm{a}$.

\begin{tabular}{|c|c|c|c|c|c|c|c|}
\hline E & $\begin{array}{c}\text { BJD(TT) } \\
\text { JD } 2400000+\end{array}$ & $\begin{array}{c}\text { Error } \\
\text { (days) }\end{array}$ & $\begin{array}{l}\text { Residual } \\
\text { (days) }\end{array}$ & $\begin{array}{r}\text { Error } \\
(\mathrm{s})\end{array}$ & $\begin{array}{r}\text { Residual } \\
\text { (s) }\end{array}$ & References & Comment \\
\hline 0 & 47344.5246635 & 0.0003500 & 0.0000290 & 30.00 & 2.51 & (1) & Reanalysed \\
\hline 2760 & 47703.5457436 & 0.0000020 & 0.0000012 & 0.17 & 0.10 & (2) & \\
\hline 2761 & 47703.6758326 & 0.0000060 & 0.0000101 & 0.52 & 0.87 & (2) & \\
\hline 2769 & 47704.7164596 & 0.0000030 & -0.0000038 & 0.26 & -0.33 & (3) & \\
\hline 2776 & 47705.6270226 & 0.0000030 & -0.0000016 & 0.26 & -0.14 & (3) & \\
\hline 2777 & 47705.7571046 & 0.0000070 & 0.0000003 & 0.60 & 0.03 & (3) & Corrected \\
\hline 2831 & 47712.7815836 & 0.0001500 & 0.0001534 & 12.96 & 13.25 & (2) & \\
\hline 2839 & 47713.8222336 & 0.0001500 & 0.0001625 & 12.96 & 14.04 & (2) & \\
\hline 7360 & 48301.9141954 & 0.0001500 & -0.0000627 & 12.96 & -5.42 & (2) & \\
\hline 28152 & 51006.5405495 & 0.0002000 & 0.0000605 & 17.28 & 5.23 & (2) & \\
\hline 30721 & 51340.7165402 & 0.0000023 & -0.0000004 & 0.20 & -0.03 & (2) & Reanalysed \\
\hline 33233 & 51667.4780058 & 0.0000960 & 0.0000041 & 8.29 & 0.35 & (4) & Reanalysed \\
\hline 38960 & 52412.4470566 & 0.0000006 & -0.0000006 & 0.05 & -0.05 & (5) & \\
\hline 38961 & 52412.5771382 & 0.0000005 & 0.0000008 & 0.04 & 0.07 & (5) & \\
\hline 38968 & 52413.4876977 & 0.0000009 & -0.0000006 & 0.08 & -0.05 & (5) & Corrected \\
\hline 38976 & 52414.5283389 & 0.0000007 & -0.0000004 & 0.06 & -0.03 & (5) & \\
\hline 38984 & 52415.5689804 & 0.0000007 & 0.0000000 & 0.06 & 0.00 & (5) & \\
\hline 41782 & 52779.5331703 & 0.0000015 & 0.0000001 & 0.13 & 0.01 & (5) & \\
\hline 41798 & 52781.6144523 & 0.0000007 & 0.0000002 & 0.06 & 0.02 & (5) & \\
\hline 41806 & 52782.6550927 & 0.0000008 & -0.0000004 & 0.07 & -0.03 & (5) & \\
\hline 41820 & 52784.4762150 & 0.0000008 & 0.0000003 & 0.07 & 0.03 & (5) & \\
\hline 44472 & 53129.4486808 & 0.0000040 & 0.0000008 & 0.35 & 0.07 & (5) & \\
\hline 44473 & 53129.5787632 & 0.0000028 & 0.0000031 & 0.24 & 0.27 & (5) & \\
\hline 44474 & 53129.7088370 & 0.0000017 & -0.0000032 & 0.15 & -0.28 & (5) & \\
\hline 44480 & 53130.4893234 & 0.0000030 & 0.0000025 & 0.26 & 0.22 & (5) & \\
\hline 49662 & 53804.5644567 & 0.0000025 & 0.0000001 & 0.22 & 0.01 & (5) & \\
\hline 49663 & 53804.6945350 & 0.0000012 & -0.0000017 & 0.10 & -0.15 & (5) & \\
\hline 49671 & 53805.7351781 & 0.0000006 & 0.0000005 & 0.05 & 0.04 & (5) & \\
\hline 53230 & 54268.6903114 & 0.0000006 & 0.0000008 & 0.05 & 0.07 & (5) & \\
\hline 53237 & 54269.6008713 & 0.0000002 & -0.0000001 & 0.02 & -0.01 & (5) & \\
\hline 56442 & 54686.5076279 & 0.0000009 & -0.0000001 & 0.08 & -0.01 & (5) & \\
\hline 58638 & 54972.1634971 & 0.0000800 & -0.0000380 & 6.91 & -3.28 & (6) & \\
\hline 58645 & 54973.0740553 & 0.0001000 & -0.0000406 & 8.64 & -3.51 & (6) & \\
\hline 58684 & 54978.1471791 & 0.0001200 & -0.0000408 & 10.37 & -3.53 & (6) & \\
\hline 58745 & 54986.0820789 & 0.0001200 & -0.0000274 & 10.37 & -2.37 & (6) & \\
\hline 58753 & 54987.1228359 & 0.0001300 & 0.0000887 & 11.23 & 7.66 & (6) & \\
\hline 58796 & 54992.7161925 & 0.0000015 & 0.0000008 & 0.13 & 0.07 & (6) & \\
\hline 60489 & 55212.9418187 & 0.0000069 & 0.0000027 & 0.60 & 0.23 & $(7,8)$ & \\
\hline 60505 & 55215.0230961 & 0.0000066 & -0.0000017 & 0.57 & -0.15 & $(7,8)$ & \\
\hline 60528 & 55218.0149380 & 0.0000043 & -0.0000024 & 0.37 & -0.21 & $(7,8)$ & \\
\hline 60735 & 55244.9415254 & 0.0000029 & 0.0000012 & 0.25 & 0.10 & $(7,8)$ & \\
\hline 60743 & 55245.9821654 & 0.0000032 & 0.0000003 & 0.28 & 0.03 & $(7,8)$ & \\
\hline 60751 & 55247.0228063 & 0.0000034 & 0.0000002 & 0.29 & 0.02 & $(7,8)$ & \\
\hline 60774 & 55250.0146469 & 0.0000034 & -0.0000018 & 0.29 & -0.16 & $(7,8)$ & \\
\hline 60927 & 55269.9169047 & 0.0000014 & -0.0000018 & 0.12 & -0.16 & $(7,9)$ & \\
\hline 60950 & 55272.9087487 & 0.0000013 & -0.0000005 & 0.11 & -0.04 & $(7,9)$ & \\
\hline 61219 & 55307.9003015 & 0.0000010 & 0.0000005 & 0.09 & 0.04 & $(7,10)$ & \\
\hline 61426 & 55334.8268834 & 0.0000018 & -0.0000025 & 0.16 & -0.22 & $(7,9)$ & \\
\hline 61440 & 55336.6480059 & 0.0000018 & -0.0000017 & 0.16 & -0.15 & $(7,9)$ & \\
\hline 61441 & 55336.7780894 & 0.0000015 & 0.0000017 & 0.13 & 0.15 & $(7,9)$ & \\
\hline 61564 & 55352.7779443 & 0.0000016 & 0.0000017 & 0.14 & 0.15 & $(7,9)$ & \\
\hline 61579 & 55354.7291448 & 0.0000009 & 0.0000004 & 0.08 & 0.03 & $(7,10)$ & \\
\hline
\end{tabular}

Notes. The published mid-eclipse times have been converted to BJD(TT) if not yet on this time standard.

References. (1) Haefner et al. (1989); ESO Msngr, 55, 61, reanalysed using up-to-date eclipse profile; (2) Haefner et al. (2004), misprint for $E=2777$ corrected, VLT trailed imaging observation $(E=30721)$ reanalysed using the original data; (3) Wood \& Marsh (1991); (4) Pigulski \& Michalska (2002), reanalysed using the original data; (5) Parsons et al. (2010b), timing for $E=38968$ corrected for misprint; (6) Qian et al. (2009); (7) this work; (8) MONET/North 1.2-m white light photometry; (9) McDonald 2.1-m photometry with Schott BG40 filter; (10) ESO NTT 3.5-m ULTRACAM Sloan $g^{\prime}$ photometry. 